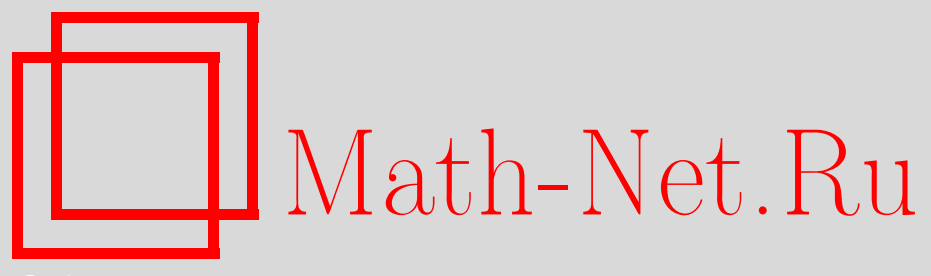

И. В. Белошапка, О неприводимых представлениях конечного веса одной дискретной нильпотентной группы, УМН, 2015, том 70, выпуск 4, 207-208

DOI: https://doi.org/10.4213/rm9674

Использование Общероссийского математического портала Math-Net.Ru подразумевает, что вы прочитали и согласны с пользовательским соглашением http: //www. mathnet.ru/rus/agreement

Параметры загрузки:

IP : 54.157 .27 .8

26 апреля 2023 г., 05:04:01

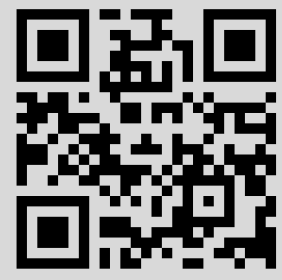




\section{О неприводимых представлениях конечного веса одной дискретной нильпотентной группы}

\section{И. В. Белошапка}

ОПредЕление 1 . Пусть $\pi: G \rightarrow \operatorname{End}(V)$ - представление группы $G$. Говорят, что представление $\pi$ является представлением с конечным весом или $\pi$ удовлетворяет условию конечности веса, если существуют такая подгруппа $H \subset G$ и такой характер $\chi$ этой подгруппы, что пространство $V(\chi, H)=\{v \in V \mid \pi(h) v=\chi(h) v \forall h \in H\}$ нетривиально и конечномерно; $H$ называется тогда весовой подгруппой представления $\pi$.

Пусть $G$ - группа унипотентных матриц размера $4 \times 4$ с целыми коэффициентами, $\pi: G \rightarrow \operatorname{End}(V)$ - ее неприводимое представление в комплексном не более чем счетномерном пространстве.

Теорема 1. Неприводимое представление $\pi$ мономиалъно, т.е. индуцировано с одномерного представления некоторой своей подгруппы, тогда и толъко тогда, когда оно является представлением с конечным весом.

Лемма 1. Пусть $V(H, \chi)$ - нетривиальное конечномерное весовое подпространство пространства представления $V, a g \in G$ - элемент группы $G$, коммутирующий со всеми элементами подгруппь $H$. Тогда существует характер $\chi^{\prime}: H^{\prime} \rightarrow \mathbb{C}^{*}$ группы $H^{\prime}=\langle H, g\rangle$ такой, что $V\left(H^{\prime}, \chi^{\prime}\right)$ нетривиалъно и конечномерно.

Утверждение следует из того, что $\pi(g) V(H, \chi)=V(H, \chi), \pi(h) \pi(g)=\pi(g) \pi(h)$ для всех $h \in H$ (т. е. $\pi\left(H^{\prime}\right)$ действует на конечномерном пространстве $\left.V(H, \chi)\right)$ и коммутирующие операторы имеют одинаковый жорданов базис для конечномерных пространств и, следовательно, имеют хотя бы один общий собственный вектор - это и дает продолжение характера $\chi$ на $H^{\prime}$.

СлЕДСтвиЕ 1. Если весовая подгруппа не содержит элемента, порождающего центр группь $G$, то такой элемент всегда можно добавить в весовую подгруппу, получал таким образом условие конечности веса относительно большей подгруппь.

Всюду далее считаем, что весовая подгруппа $H$ содержит центр группы $G$. Имеем следующую точную последовательность:

$$
1 \rightarrow[G, G] \rightarrow G \stackrel{\mathrm{pr}}{\longrightarrow} G /[G, G] \rightarrow 1 .
$$

Группа $G$ является нильпотентной группой класса нильпотентности 3. Для всякой подгруппы $H \subset G$ имеем точную последовательность

$$
1 \rightarrow[G, G] \cap H \rightarrow H \rightarrow \operatorname{pr}(H) \rightarrow 1 .
$$

Положим $m=\operatorname{rk}_{\mathbb{Q}} \operatorname{pr}(H) \otimes \mathbb{Q}, n=\operatorname{rk}_{\mathbb{Q}}[G, G] \cap H \otimes \mathbb{Q}$.

ПреДЛОЖЕНИЕ 1 [1]. Пусть $\pi$ - неприводимое представление нилъпотентной дискретной группы $G$ и существует нормальная подгруппа $H \triangleleft G$, относительно которой выполняется условие конечности веса. Тогда $\pi$ мономиально.

Теорема 2 [2]. Пусть $\pi$ - неприводимое конечномерное представление нильпотентной дискретной группы $G$. Тогда $\pi$ мономиально.

Исследование выполнено за счет гранта Российского научного фонда (проект № 14-50-00005).

DOI: $10.4213 / \mathrm{rm} 9674$ 
Необходимость в теореме 1 следует из критерия Макки и двойственности Фробениуса [3], [4; гл. I.5], с помощью которых вычисляются $\operatorname{Hom}^{G}\left(\operatorname{ind}_{H}^{G}(\chi), \operatorname{ind}_{H}^{G}(\chi)\right)$; а именно, из неприводимости $\operatorname{ind}_{H}^{G}(\chi)=\pi$ следует, что характер $\chi$ однократно входит в представление $\pi$. Докажем теперь достаточность в утверждении теоремы 1 , разбирая различные ранги весовых подгрупп $(m, n), 0 \leqslant m \leqslant 3,0 \leqslant n \leqslant 3$.

- Ранги $(0,1) u(3,3)$. Условие конечности веса относительно подгрупп таких рангов означает, что представление $\pi=\operatorname{ind}_{H}^{G}(\chi)$ конечномерно, а для конечномерных представлений утверждение выполняется по теореме 2.

- Ранги $(1,1),(0,2)$ u $(0,3)$. Ранг весовой подгруппы в этих случаях может быть увеличен добавлением коммутирующего элемента, который можно добавить с сохранением весового условия по теореме 1.

- Ранги $(1,2),(1,3),(2,1),(2,2)$ u $(2,3)$. Для подгрупп таких рангов проверяем условие $\operatorname{Hom}^{G}\left(\operatorname{ind}_{H}^{G}(\chi), \operatorname{ind}_{H}^{G}(\chi)\right)=\mathbb{C}$, и если оно выполняется, доказываем от противного неприводимость представления $\pi=\operatorname{ind}_{H}^{G}(\chi) . \operatorname{Ecли~же~} \operatorname{Hom}^{G}\left(\operatorname{ind}_{H}^{G}(\chi), \operatorname{ind}_{H}^{G}(\chi)\right)$ нетривиально, то можно показать, что существуют элементы группы $G$, коммутирующие со всеми элементами весовой подгруппы, мы их добавляем по теореме 1 и либо после этого получаем нормальную весовую подгруппу, и тогда утверждение верно по предложению 1 , либо получаем представление с $\operatorname{Hom}^{G}\left(\operatorname{ind}_{H^{\prime}}^{G}\left(\chi^{\prime}\right), \operatorname{ind}_{H^{\prime}}^{G}\left(\chi^{\prime}\right)\right)=\mathbb{C}$, неприводимость которого доказываем от противного. Это делается так: можно явно выписать базис пространства представления $\operatorname{ind}_{H^{\prime}}^{G}\left(\chi^{\prime}\right)$, посмотреть на действие группы $G$ и доказать отсутствие нетривиальных инвариантных подпространств.

- Ранги $(3,1)$ u $(3,2)$. Легко проверить, что подгрупп с такими рангами в $G$ нет (три образующих с ненулевой проекцией в $G /[G, G]$ обязательно дают ранг 2 в $[G, G] / Z(G))$.

Таким образом, мы доказали следующую теорему.

Теорема 3. (а) Пусть $H$ - подгруппа группы $G$ унипотентных матрии, размера $4 \times 4$ с иелыми коэффициентами и $\operatorname{Hom}^{G}\left(\operatorname{ind}_{H}^{G}(\chi), \operatorname{ind}_{H}^{G}(\chi)\right)=\mathbb{C}$, тогда представление $\operatorname{ind}_{H}^{G}(\chi)$ неприводимо.

(b) Если $\pi$ - неприводимое представление конечного веса, то среди его весовых подгрупп всегда найдется подгруппа одного из следующих рангов: $(0,1),(1,2),(1,3)$, $(2,1),(3,3)$.

(c) Если т бесконечномерно, то этот список короче: $(1,2),(1,3),(2,1),(2,2),(2,3)$.

\section{Список литературы}

[1] С. А. Арналь, А.Н. Паршин, Матем. заметки, 92:3 (2012), 323-330; англ. пер.: S. A. Arnal', A. N. Parshin, Math. Notes, 92:3 (2012), 295-301. [2] I. D. Brown, Pacific J. Math., 45:1 (1973), 13-26. [3] P. C. Kutzko, Proc. Amer. Math. Soc., 64 (1977), 173-175. [4] M.-F. Vignéras, Représentations $l$-modulaires d'un groupe réductif $p$-adique avec $l \neq p$, Progr. Math., 137, Birkhäuser Boston, Inc., Boston, MA, 1996, xviii+233 pp.

Иулия Валериевна Белошапка

(Iuliya V. Beloshapka)

Математический институт им. В. А. Стеклова

Российской академии наук

E-mail: i-beloshapka@yandex.ru
Представлено А. Г. Сергеевым Принято редколлегией 30.06 .2015 